\title{
SIMULATION USING FEM FOR DESIGN PMDC MOTORS
}

\author{
Oliveira Lucas ${ }^{1}$, Carvalho Patrick ${ }^{2}$ e Giesbrecht Mateus ${ }^{3}$ \\ ${ }^{1}$ Valeo Sistemas Automotivos \\ ${ }^{2}$ Valeo Sistemas Automotivos \\ ${ }^{3}$ Universidade Estadual de Campinas UNICAMP \\ E-mails: lucas.oliveira@valeo.com, patrick.carvalho@,valeo.com, \\ mateus@fee.unicamp.br
}

\begin{abstract}
The growth of electrical vehicles market has increased the demand for permanent magnet direct current motors (PMDC). The design of PMDC motors involves a great amount of time, therefore assertive ways to design it can save time and money. This work demonstrates how to make use of electromagnet flux simulation in order to forecast direct current motors performance. When the flux simulation was compared to the results of tests applied on a prototype it was shown that the simulation is able to forecast prototype performance with an error of $6 \%$. Then it is demonstrated that simulations can be of good use for speed up design process, ensuring agility to design process that electrical vehicles market requires.
\end{abstract}

\section{INTRODUCTION}

Automotive market becomes more competitive every year, all cost reduction and innovation means improvement on competitiveness. Then in current times of Industry 4.0 [1], methods for design and prediction of products performance are mostly required. Great importance is given to know the performance of a product beforehand, at rate that it impacts directly on the cost of the project. Besides that, fast ways to test several behaviors are of great use for engineers involved in projects of innovative design, therefore methods and tools that simplify those tasks are welcome.

The use of electric motors for vehicles accessories has become common, as for example to move car windows, assist the steering wheel and many other applications that are designed every day to simplify and improve the user experience. However to design such electric motors, the power supply available on application vehicle, which is DC, must be taken into account. Therefore DC electric motors are the most common application.

A special case of those motors are the Permanent Magnet Direct Current (PMDC) motors. Those machines are important because of its reduced dimension and cost, so it has become widely used to operate electric windows, windshield wipers, starter motors and many others. This article explores the application of flux density estimative by finite element method (FEM) to design PMDC motors. Several other methods are available for PMDC [2][3]. Meanwhile, the use of simulation (FEM) simplifies the 
computation task and reduces the calculation error, as well as there are several effects that are better approximated with a FEM simulation This work proposes to compare the results of a simulation with the results acquired in a prototype tested in the laboratory. A starter motor with PMCD design will be used as scope.

\section{PRINCIPLE OF PMDC MOTOR AND STRUCTURE}

The PMDC motor is a machine composed by a stator of permanent magnets and a rotor of armature windings, the inversion of armature current that induces the rotation are caused by brushes and commutator, the main effects that helm the functionality of a PMDC motors are described by the Ampere law (1) and Lorentz force (2) [3].

$$
\begin{aligned}
& \oint B \cdot d l=I \mu \\
& F=I(L X B)
\end{aligned}
$$

Deriving from Lorentz force due the effect caused by the several windings on armature, the resultant of machine torque is given by the following equation (3), where $I_{A}$ is the current passing thorough the armature windings and $\varnothing$ is the electromagnet flux that crosses the windings.

$$
T=K_{a} \varnothing I_{a}
$$

The $K_{a}$ is one construction constant derived by the elements of motor construction. $K_{a}$ is given by equation 4 , in the case evaluated here $K_{a}$ is equal to 47.746 .

$$
K_{a}=\frac{Z P}{2 \pi a}
$$

Where $Z$ is the number of conductors in the armature winding, $P$ is the number of poles, $a$ is the number of current paths through the armature and is correlated to the type of windings construction. For the PMDC motor evaluated a value is equal to 2, since the armature winding is of the wave type.

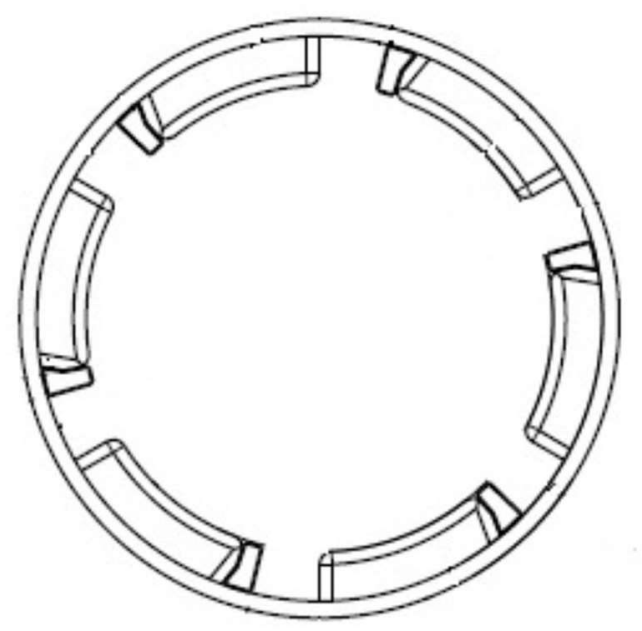

Fig. 1. Illustration of stator, front view 
The flux $(\varnothing)$ value on equation 2 is correlated to the Ampere Law and is associated to magnet field created by the magnet poles on stator of motor. The equation (5) shows the relationship between magnet flux and flux density.

$$
\emptyset=B A
$$

Where A is the area of the cross section of the flux path. The PMDC motor evaluated presents a stator with 6 magnetic poles and 6 auxiliary poles, the structure is presented in Fig 1. The armature has 25 slots according to the Fig 2 and has 4 wires per slot.

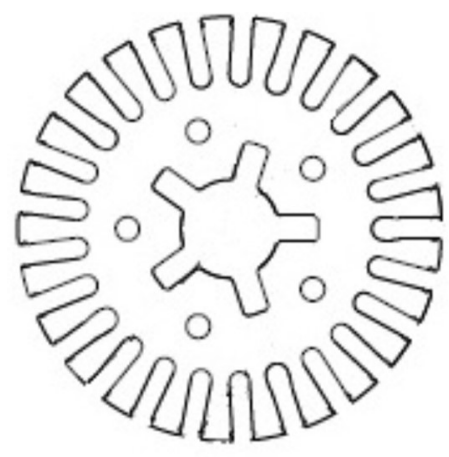

Fig. 2. Illustration of rotor, front view

\section{COMPUTATIONAL SIMULATION FOR MAGNECT PROBLEM SOLVING}

Problems in the field of physics are usually possible to be expressed in partial differential equations. The differential equations that held the electromagnetism phenomena were first presented by the Scottish mathematician James Clerk Maxwell. Partial differential equations are often very complicated to be solved by analytical methods, so numerical methods capable of solving this type of equation have become of great necessity.

Finite Element Method (FEM) is basically a method capable of solving a differential equation numerically without the need to change the formulation of the problem and the computational code to obtain the solution. The method was first developed for problems related to structural calculation, however today it is used in other areas such as fluid dynamics, thermodynamics and magnetism.

The method is based on the discretization of the problem, where the problem domain is broken into small structures such as hexahedron, tetrahedron or triangle, then a mesh is formed. This mesh can have $\mathrm{N}$ elements, where the quantity is relatively proportional to the refinement of the discretization and inversely proportional to the computational time to solve the problem.

As can be seen in Fig 3, generally for a good accuracy in problem solution, a large discretization of structure must be done, in order to ensure a good cover over points of interest on structure such as laterals, points of greater field transient. A greater amount of elements makes the problem result richer. Some software's work with a mesh optimization process or self-adaptive mesh, so that a more precise solution can be obtained with fewer elements. 


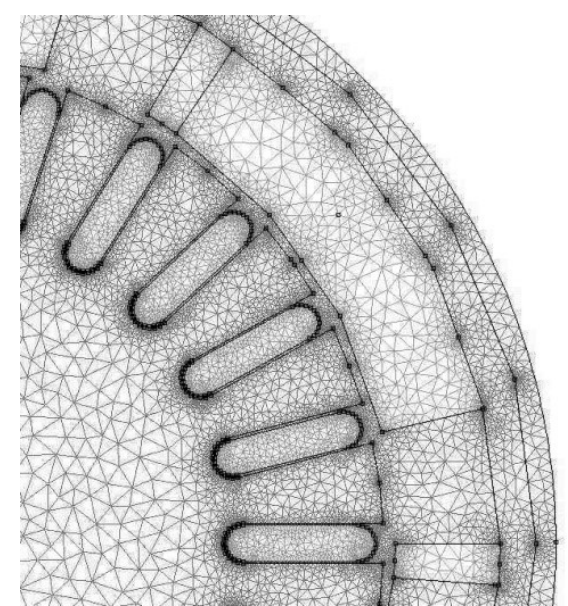

Fig.3 - 2D Triangle Mesh to Geometric

After the discretization of the problem domain, that has a physical origin, the problem is approximated by polynomial equations, so that it can be treated as a matrix for the solution to be executed by computer. Due to the problem complexity, it can be solved only on computers.

The finite element method can be applied directly via mathematical software. The most common today is the use of specialized software designed to this type of task. Commercially there are several options like Flux2D, JMAG, ANSOFT Maxwell, FEMM, among others.

The problem discussed in this paper is related to the magnetostatic scope. In order to solve the proposed problem the FEMM Software version 4.2 was used. This software provides full documentation [4]. The software features a graphical CAD interface. The interface allows the insertion of structures that will be the focus of the solution.

In the interface it is possible to assign materials and excitations, to the drawn geometries. The materials are usually available in a library, but can be included directly by the user. The solution is given in the form of spectral or rectangular graphs, it is also possible to calculate magnitudes from the structures and fields.

Solver Magnetostatic solves problem containing linear and non-linear materials, where a static magnetic field is originated by a continuous current flowing in a coil or due to a permanent magnet. To solve the problem, the software solves the following differential equations:

$$
\nabla \times H=J
$$

Equation (6) is the Maxwell equation in its static model for the Ampere law. However, the Magnetostatic Solver solves the problem in terms of the Magnetic Vector Potential $A$, in this way we see the relation between the magnetic flux density and the Magnetic Vector Potential is given by the following equation:

$$
B=\nabla \times A
$$

Moreover, it is necessary to use the constitutive relation between Magnetic Flux Density and Magnetic Flux Intensity, given in the linear and isotropic media by the following equation: 


$$
H=\frac{1}{\mu_{0} \mu_{r}}
$$

Therefore, replacing equations (7) and (8) in equation (6) we have:

$$
J=\nabla x\left(\frac{1}{\mu_{0} \mu_{r}}(\nabla x A)\right)
$$

The software solves the equation 9 to the domain, allowing the determination of the desired fields.

\section{MATERIAL AND FEM SIMULATION SET-UP}

The materials of the PMDC motor model must be assigned to match with the real world PMDC motor. This stage has great importance, at rate that any difference from PMDC motor model to the real PMDC motor can produce a poor result of simulation. The materials for simulation were assigned according to the Table I.

TABLE I - Material Assignment

\begin{tabular}{llr}
\hline Part & Material & $\begin{array}{r}\text { Magnetic permeability } \\
(\mu)\end{array}$ \\
\hline Yoke & Steel 1018 & $2.5 \cdot e^{-2} H / m$ \\
Auxiliary pole & Steel 1018 & $2.5 \cdot e^{-2} H / m$ \\
Armature core & Steel 1018 & $2.5 \cdot e^{-2} H / m$ \\
215 & Copper & $1.256 \cdot e^{-6} H / m$ \\
310 & Ferrite & $2.5 \cdot e^{-6} H / m$ \\
Air gap & Air & $4 \pi \cdot e^{-6} H / m$ \\
\hline
\end{tabular}

The materials are assigned to the PMDC motor according to construction illustrated on Fig 4 . The ferrite material has the following parameters: $B_{r}=0: 4 \mathrm{~T}$ and $H_{c}=302 \mathrm{KA} / \mathrm{m}$.

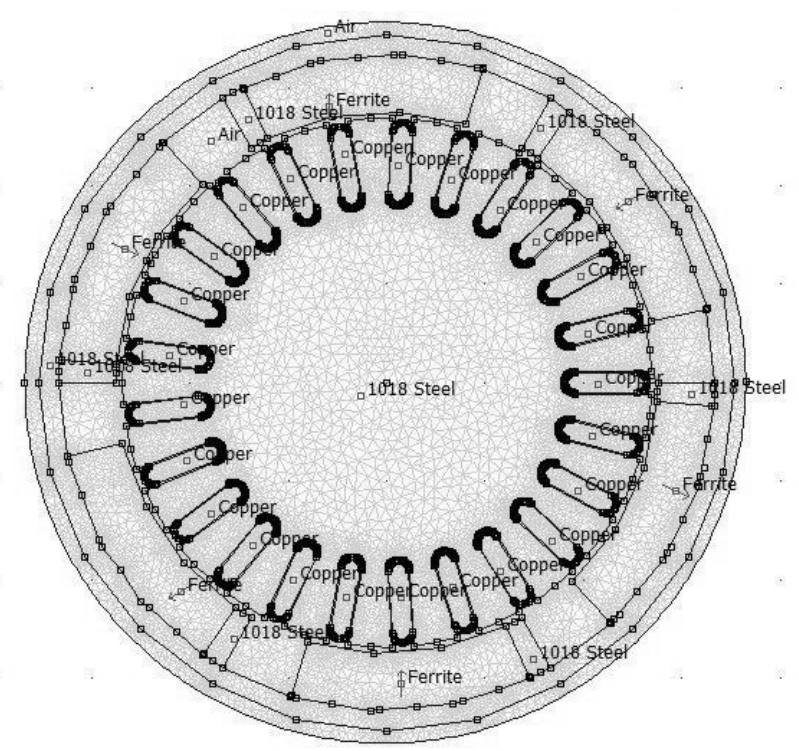

Fig. 4. PMDC motor construction material assigned 


\section{RESULTS}

As result of simulation was obtained the following magnetic flux density, showed in Fig 5.

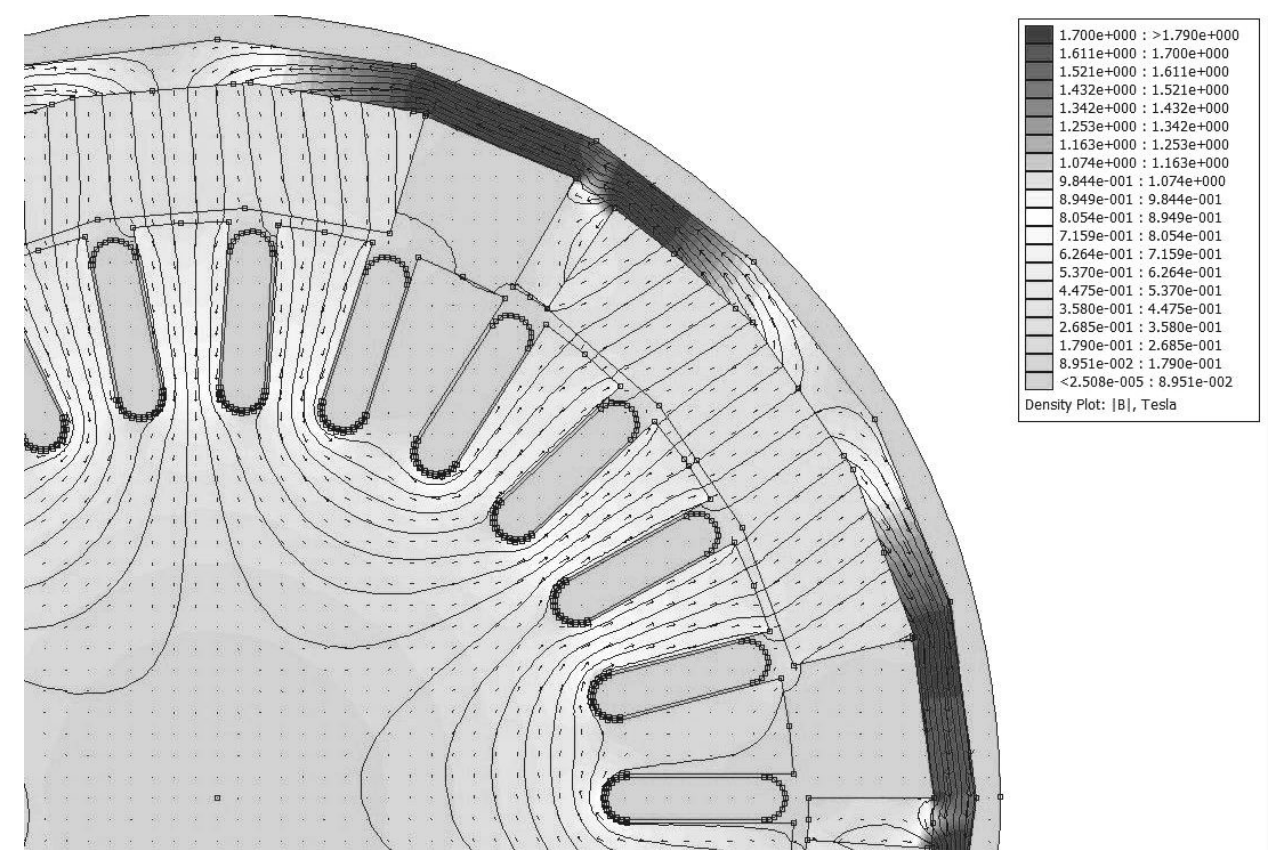

Fig. 5. Magnetic flux density distribution on PMDC motor structure

The Fig 6 shows the graph of magnetic flux density for the entire perimeter of air gap. It is possible to observe the peaks and valleys corresponding to the interlope region and the slot region.

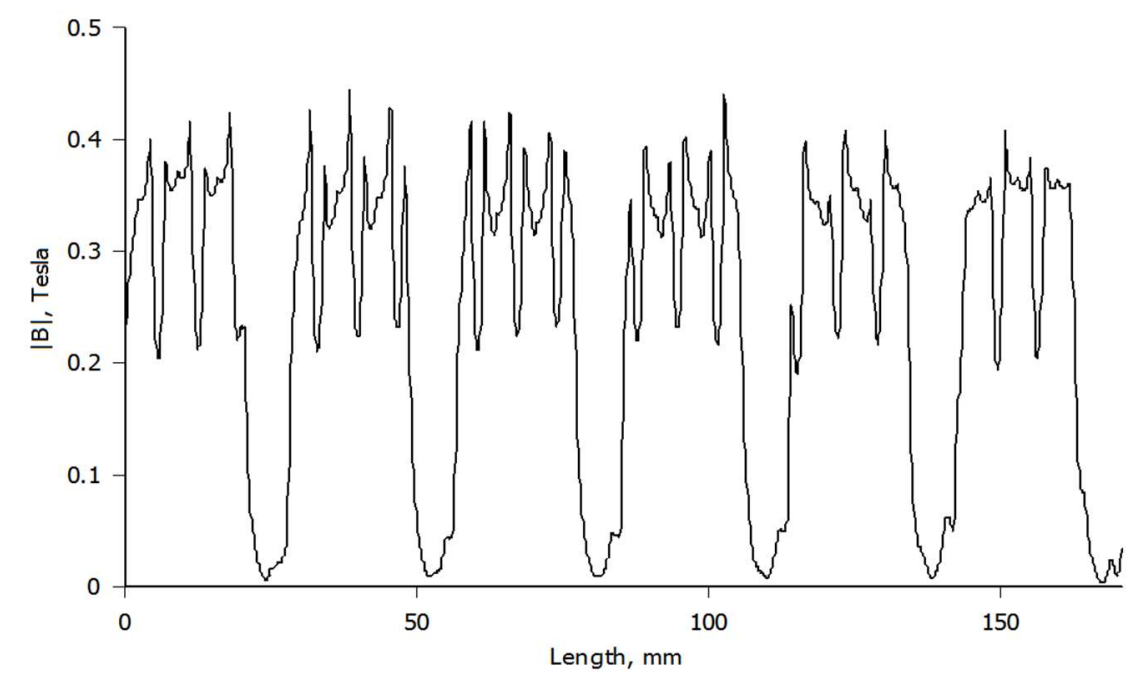

Fig. 6. Magnetic flux density distribution across PMDC motor air gap perimeter

In Fig 6 is presented the value of magnetic flux density distributed over the magnetic poles. The average value of magnetic flux density over the magnetic pole is calculated. Therefore the value, according to equation (5), is multiplied by the height of magnetic poles to obtain flux density $\emptyset$. 
Applying the values of $\varnothing$ and $\mathrm{Ka}$ already obtained on equation (3), it is possible to calculate the torque developed on the PMDC motor for several conditions of current. To calculate the torque that is available to the shaft, it is necessary to discount the mechanical losses. This consideration has to be done because the equation (3) gives the developed torque and it is needed to be compared to the mechanical torque measured on the test done with prototypes.

For this PMDC motor, a value of 40 A was discounted from the values obtained in the simulation to represent the losses (no load current). This approximation will be done due to the losses related to the armature reaction effect and to the mechanical losses.

In the Fig 7, a comparison between the simulation results and the prototype tested on laboratory test bench is shown. Also the comparative results demonstrate on Table II shows an error lower than $6 \%$.

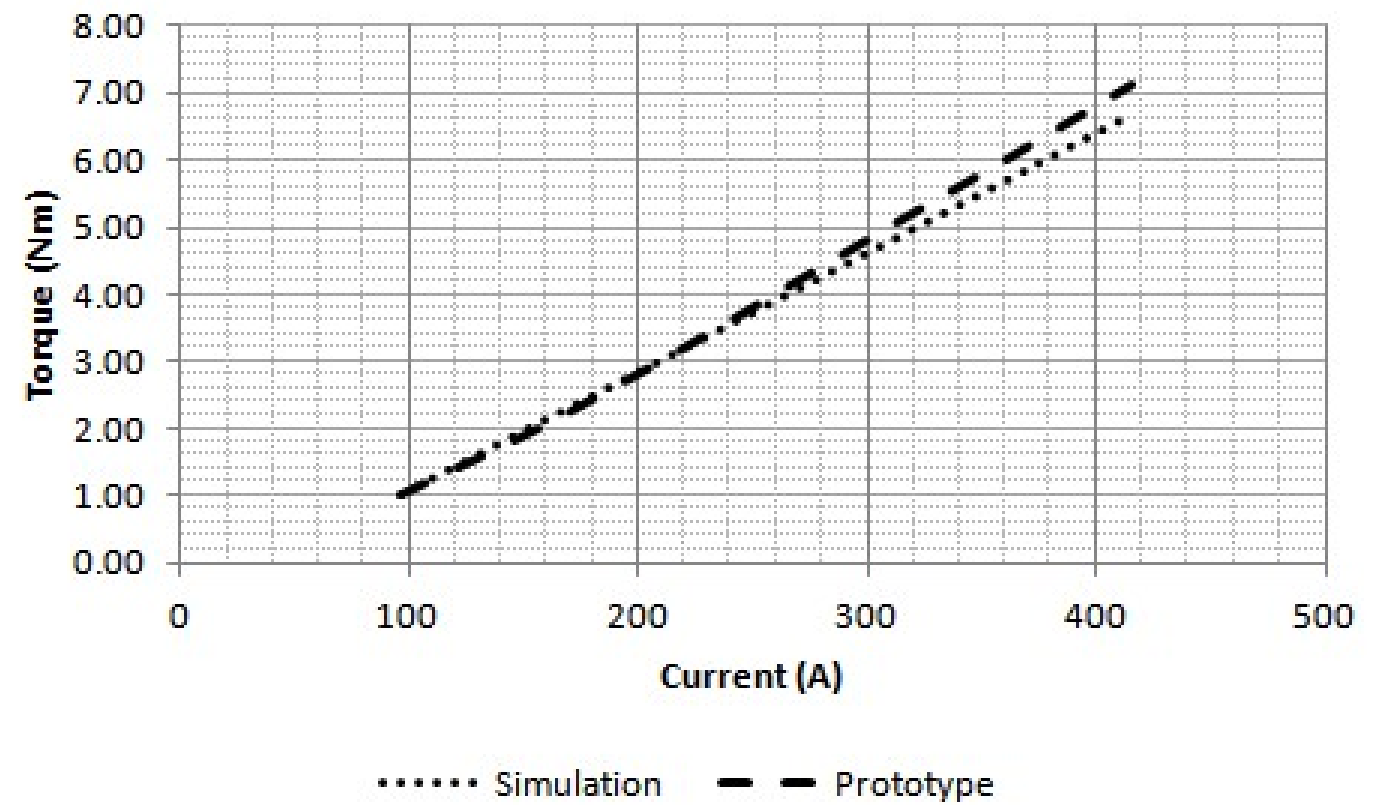

Fig.7. Comparative results between simulation and prototype tested on a laboratory test bench

TABLEII - Comparative Results of simulations and prototype tests

\begin{tabular}{lrrr}
\hline $\begin{array}{l}\text { Current } \\
\text { applied } \\
(\mathrm{A})\end{array}$ & $\begin{array}{r}\text { Torque for } \\
\text { Simulation } \\
(\mathrm{Nm})\end{array}$ & $\begin{array}{r}\text { Torque for } \\
\text { prototype tests }(\mathrm{Nm})\end{array}$ & $\begin{array}{r}\text { Module of } \\
\text { prediction error } \\
(\%)\end{array}$ \\
\hline 96 & 1.00 & 1.0 & $0.92 \%$ \\
157 & 2.08 & 2.0 & $4.46 \%$ \\
183 & 2.54 & 2.5 & $1.98 \%$ \\
215 & 3.11 & 3.1 & $0.52 \%$ \\
310 & 4.79 & 5.0 & $4.04 \%$ \\
356 & 5.60 & 5.9 & $4.87 \%$ \\
416 & 6.67 & 7.1 & $5.99 \%$ \\
\hline
\end{tabular}




\section{CONCLUSION}

This paper presents the main points on how to make use of magnetic flux simulation to forecast electrical characteristics of PMDC machines, revealing that simulation predicts the electrical characteristics of machines with an error lower than $6 \%$ compared to prototype tested on test bench. It demonstrates that simulation is a powerful tool to reduce time and resources expend on design process. It is even possible to improve assertiveness by the mesh refinement, geometrical structure and losses. This work also give bases for future works related to the mathematical optimization, at rate that is possible to create a virtual set of machines by use of FEM to be submitted to optimization algorithm, or even to validate new machine design concepts created by optimization method.

\section{REFERENCES}

[1] HERMANN, M; PENTEK T; and OTTO B; Design Principles for Industries 4.0 Scenarios, 2016 49th Hawaii International Conference on System Sciences.

[2] WEST, J; PMDC motors with series field characteristics, Proc. IEE Colloquium Permanent Magnet Machines, 1988, p. 5.

[4] SEN, P; Principles of Electric machines and power electronics, 2th ed. John Wiley \& Sons, 1997 p. 121.

[5] MEEKER, D; FEMM Reference Manual, 2018. Available: http://www.femm.info /wiki/Documentation. [Accessed: 11- Mai- 2018]. 